\title{
AGO Recommendations for Diagnosis and Treatment of Patients with Early Breast Cancer: Update 2013
}

\author{
Anton Scharl ${ }^{\mathrm{a}}$ Christoph Thomssen ${ }^{\mathrm{b}} \quad$ Nadia Harbeck $^{\mathrm{c}} \quad$ Volkmar Müller $^{\mathrm{d}}$; on behalf of the AGO \\ Breast Committee* \\ aFrauenklinik, Martin-Luther Universität Halle/Saale, b Frauenklinik, Klinikum Amberg, 'Brustzentrum, Frauenklinik, Universität München, \\ dKlinik für Gynäkologie, Universitätsklinikum Hamburg-Eppendorf, Hamburg, Germany
}

\section{Introduction}

For the last 12 years, the Breast Committee of the Arbeitsgemeinschaft Gynäkologische Onkologie (German Gynaecological Oncology Group, AGO) has been preparing and updating evidence-based recommendations for the diagnosis and treatment of patients with early and metastatic breast cancer. The AGO Breast Committee consists of 43 gynaecological oncologists specialized in breast cancer and interdisciplinary members specialized in pathology, radiological diagnostics, medical oncology and radiotherapy. The update is performed according to documented rules by thoroughly reviewing and scoring chapter by chapter the recent publications for their scientific validity (Oxford Level of Evidence, LoE; www.cebm.net [1]) and clinical relevance (AGO Grades of Recommendation; table 1). Here we present the 2013 update of these guidelines focussing on the modifications that were performed this year. The full version of the update is available online as a PDF file in an English and a German version [2].

\section{Prognostic and Predictive Factors}

Currently, the indication for adjuvant chemotherapy is mainly driven by prognosis and to a much lesser extent by prediction. Since the publication of the molecular classification of breast cancer, the role of classical pathology and immunohistochemistry (IHC) has been questioned as a sole instrument for adju- vant decision making. According to ASCO-CAP guidelines, discordances for central versus local immunohistochemical staining of hormone receptors (HR) and human epidermal growth factor receptor 2 (HER2) are reported in about $20 \%$, major discrepancies in grading for $40 \%$ [3-5]. Furthermore, in 2012, Mirror trialists reported an upgrade of $22 \%$ of pN0 cases to pN1 in central pathology [6]. In the context of these data, and because of the lack of consideration of HER2 overexpression as a prognostic and predictive factor, the AGO guidelines have downgraded the available version 8.0 of Adjuvant! online ( $\mathrm{LoE} 2 \mathrm{bB}$; $\mathrm{AGO}+/-$ ). Considering immunohistochemical tumour markers, $\mathrm{Ki}-67$ is a reliable prognostic factor especially after neoadjuvant chemotherapy (NACT)/ short-term endocrine treatment. Data for prediction of chemotherapy outcome are less convincing. The committee nevertheless recommends the clinical use of $\mathrm{Ki}-67$ under the prerequisite of meticulous quality control ( $\mathrm{LoE} 1 \mathrm{aA} ; \mathrm{AGO}+)$. As long as nationwide standardization and quality assurance are not implemented, cut-off levels cannot be reliably defined for routine use.

UPA/PAI was tested in prospective trials and is suggested as a reliable prognostic marker and a predictive marker for the usefulness of chemotherapy in N0 cases (LoE 1aA; $\mathrm{AGO}+$ ).

New molecular tools (mRNA, DNA level) have the advantage of higher accuracy, reproducibility and lower interobserver variability compared to IHC. To allow for adequate evaluation of available molecular markers/genomic signatures,

\footnotetext{
* Members of the 'AGO Breast Committee' in alphabetical order: Ute-Susan Albert, Marburg; Ingo Bauerfeind, Landshut; Joachim Bischoff, Magdeburg; Jens Uwe Blohmer, Berlin; Klaus Brunnert, Osnabrück; Peter Dall, Lüneburg; Ingo J. Diel, Mannheim; Tanja Fehm, Düsseldorf; Nikos Fersis, Chemnitz; Michael Friedrich, Krefeld; Kay Friedrichs, Hamburg; Bernd Gerber, Rostock; Volker Hanf, Fürth; Nadia Harbeck, München; Jens Huober, St. Gallen; Christian Jackisch, Offenbach;Wolfgang Janni, Ulm (Co-Chair); Walter Jonat, Kiel (DKH); Hans H. Kreipe, Hannover (DGP); Sherko Kümmel, Essen; Sibylle Loibl, Neu-Isenburg; Hans-Joachim Lück, Hannover; Michael Lux, Erlangen; Nicolai Maass, Aachen; Gunter von Minckwitz, Neu-Isenburg; Volker Möbus, Frankfurt; Christoph Mundhenke, Kiel; Volkmar Müller, Hamburg; Ulrike Nitz, Mönchengladbach; Mahdi Rezai, Düsseldorf; Achim Rody, Lübeck; Anton Scharl, Amberg (Chair); Rita Schmutzler, Köln; Marcus Schmidt, Mainz; Andreas Schneeweiß, Heidelberg (AIO); Ingrid Schreer, Kiel (DGS); Florian Schütz, Heidelberg; Peter Sinn, Heidelberg (Pathologie); Erich F. Solomayer, Homburg; Rainer Souchon, Tübingen (ARO); Elmar Stickeler, Freiburg; Christoph Thomssen, Halle (Saale); Michael Untch, Berlin
}

\section{KARGER}

Fax +497614520714

Information@Karger.com

www.karger.com
(C) 2013 S. Karger GmbH, Freiburg $1661-3791 / 13 / 0083-0174 \$ 38.00 / 0$

Accessible online at:

www.karger.com/brc
Prof. Dr. Anton Scharl

Frauenklinik

Klinikum St. Marien

92224 Amberg, Germany

scharl.anton@klinikum-amberg.de 
Table 1. AGO grades of recommendation

++ This investigation or therapeutic intervention is highly beneficial for patients, can be recommended without restriction, and should be performed.

$+\quad$ This investigation or therapeutic intervention is of limited benefit for patients and can be performed.

+/- This investigation or therapeutic intervention has not shown benefit for patients and may be performed only in individual cases. According to current knowledge a general recommendation cannot be given.

- This investigation or therapeutic intervention can be of disadvantage for patients and might not be performed.

-/- This investigation or therapeutic intervention is of clear disadvantage for patients and should be avoided or omitted in any case.

the AGO Breast Committee valued prospective-retrospective evidence, generated by retrospective analyses using archived tissue from prospective trials, to LoE IB as proposed by Simon et al. in 2009 [7]. Validated molecular signatures may be used in individual cases in which classical prognostic factors provide contradictory results; however, a general recommendation cannot be given for lack of prospective data (LoE 2bB; AGO+/-). The largest prospective-retrospective body of evidence exists for Oncotype DX ${ }^{\circledR}$ (Genomic Health Inc., Redwood City, CA, USA) (LoE IB, prognostic and predictive for chemotherapy) in $\mathrm{HR}+\mathrm{N} 0-1$ breast cancer $[8$, 9]. Endopredict ${ }^{\circledR}$ (Sividon Diagnostics GmbH, Cologne, Germany) (LoE IB for prognosis) was evaluated in HRpositive postmenopausal patients receiving endocrine therapy only and cannot be used for prediction of chemotherapy outcome [10]. Mammaprint ${ }^{\circledR}$ (Agendia BV, Amsterdam, Netherlands) has been evaluated in N0-1 breast cancer (LoE IIC for prognosis) [11]. Additionally, PAM50, a gene expression signature which reproduces molecular subtypes (LoE IIB for prognosis), will soon be commercially available in Germany [12].

\section{Ductal Carcinoma In Situ}

About 74,000 women are diagnosed with primary breast cancer in Germany every year [13]. This rate has increased over the last years after the introduction of a mammography screening program in 2006. This high incidence underlines the relevance of an interdisciplinary diagnostic and therapeutic management.

For the pretherapeutic assessment of suspicious lesions (BIRADS IV), stereotactic core needle biopsy or vacuumassisted biopsy are recommended ( $\mathrm{LoE} 2 \mathrm{bB} ; \mathrm{AGO}++)$. If the lesion is completely removed in the course of the biopsy, a marker clip should be left at the biopsy site to mark the exact location of the lesion ( $\mathrm{LoE} 5 \mathrm{D}$; AGO++). Moreover, a clinical examination should be performed.

When planning the type of surgery, it should be considered that patients diagnosed with ductal carcinoma in situ (DCIS) and a palpable mass have a significantly higher potential for occult invasion (26\%), multicentricity and local recurrence. Breast-conserving surgery (BCS) is often possible, but in the case of tumour-free margins of less than $2 \mathrm{~mm}$ re-excision is recommended ( $\mathrm{LoE} 2 \mathrm{C}$; $\mathrm{AGO}+$ ). Mastectomy should be considered in the case of large lesions (confirmed by multiple biopsies) or positive margins after re-excision (LoE 2aB; $\mathrm{AGO}++)$. When mastectomy is required, a simultaneous sentinel node excision (SNE) should be performed, as SNE after mastectomy is not feasible if an invasive component is diagnosed in the mastectomy tissue ( $\mathrm{LoE} 3 \mathrm{bB} ; \mathrm{AGO}+$ ). In the case of BCS and a large DCIS $(>5 \mathrm{~cm})$ or DCIS $\geq 2.5 \mathrm{~cm}$ of high-grade and/or with comedonecrosis, SNE should be discussed individually ( $\mathrm{LoE} 3 \mathrm{bB}$; $\mathrm{AGO}+-$ ). DCIS in male patients should be treated with mastectomy and SNE (LoE $5 \mathrm{D} ; \mathrm{AGO}+$ ).

Regarding radio-oncological aspects, new data confirmed that there is no patient subset - not even a low-risk subgroup - that does not benefit from radiotherapy after BCS in terms of improved local tumour control (LoE 1a) [14]. As for radiation therapy after BCS in patients with invasive breast cancer, hypofractionated radiotherapy might be as safe and effective as normofractionated (i.e. standard) radiotherapy. This is now being tested in a randomised phase III study of radiodoses and fractionation schedules in non-low-risk DCIS of the breast to improve time to recurrence (TROG 07.01; www.anzctr.org.au).

Concerning the use of tamoxifen after surgery with or without radiotherapy of DCIS, a Cochrane meta-analysis was published in 2012 [15]. 2 randomized controlled trials were included involving 3,375 women. Tamoxifen reduced the risk of recurrence of ipsilateral DCIS (hazard ratio (HR) 0.75; $95 \%$ confidence interval (CI) $0.61-0.92$ ) and contralateral DCIS (relative risk (RR) $0.50 ; 95 \%$ CI $0.28-0.87$ ). There was a trend towards decreased ipsilateral invasive cancer (HR $0.79 ; 95 \%$ CI $0.62-1.01)$ and reduced contralateral invasive cancer (RR 0.57; 95\% CI 0.39-0.83). The number needed to treat to have a protective effect against all breast events was 15. No reliable number needed to harm could be calculated. Moreover, it was not clear how patient characteristics (e.g. menopausal status, age and tumour oestrogen receptor (ER) status) affected or predicted response to tamoxifen. There was no evidence for a difference in mortality (RR 1.11; 95\% CI 0.89-1.39). Therefore tamoxifen is an option after considering risk and benefit ( $\mathrm{LoE} 1 \mathrm{aA} ; \mathrm{AGO}+)$.

\section{Neoadjuvant Chemotherapy}

In the past year, no practice-changing data concerning NACT have been presented. Thus, only minor modifications have been made in the 2013 AGO recommendations. In general, 
NACT is a therapy option if similar postoperative adjuvant chemotherapy is indicated (LoE $1 \mathrm{bA} ; \mathrm{AGO}+$ ). In particular, in patient subgroups where pathologic complete response (pCR) is associated with improved survival, such as triplenegative and HER2-positive, NACT (plus targeted therapy) should be the preferred therapeutic approach (AGO+). In triple-negative breast cancer, platinum salts, appearing promising as active agents in BRCA mutation carriers, should be given preferably within the context of clinical trials. A recent trial failed to demonstrate additional benefit [16]. Results from further trials are awaited (e.g. ADAPT, GeparSixto). Response-guided treatment has proven to be beneficial within the Gepartrio trial [17]. Consequently, in the case of response after 2 cycles of DAC (docetaxel, doxorubicin, cyclophosphamide) in HR-positive breast cancer, a total of 8 instead of 6 cycles of DAC may be considered (LoE 2bC; $\mathrm{AGO}+$ ). In the case of non-response after 2 cycles of DAC, continuation of neoadjuvant systemic therapy with a non-cross-resistant regimen, such as $4 \times \mathrm{NX}$ (vinorelbine and capecitabine) may be beneficial ( $\mathrm{LoE} 2 \mathrm{bB} ; \mathrm{AGO}+$ ).

With regard to endocrine neoadjuvant therapy, prognostic factors (such as quantitative ER expression, level of Ki-67, $\mathrm{N}$ status or T status, e.g. PEPI score) can be assessed during and after NACT (LoE 1bB; AGO+).

Indications for mastectomy after NACT are: positive margins after repeated excisions (LoE 3bC; $\mathrm{AGO}++$ ), radiotherapy not feasible ( $\mathrm{LoE} 5 \mathrm{D} ; \mathrm{AGO}++$ ), and inflammatory breast cancer in the case of only clinical CR (LoE 2bC; $\mathrm{AGO}+$ ). In inflammatory breast cancer with pCR after NACT, BCS may be discussed with the patient $(\mathrm{AGO}+/-)$ as an individual option mentioning the scarce data base for such an approach. Similarly, multicentric lesions and large tumours (cT4a-c) are only relative indications for mastectomy after NACT (AGO+/-).

\section{Breast Cancer Surgery - Oncological Aspects}

Two studies concerned with the role of sentinel node biopsy (SNB) before and after NACT were presented and published at the San Antonio Breast Cancer Symposium 2012. The German SENTINA trial is a 4-arm prospective multicentre cohort study designed to evaluate a specific algorithm for the timing of a standardized SNB procedure [18]. In addition to the clinical examination, all patients underwent an axillary ultrasound before starting NACT. In $20 \%$ of clinically nodepositive patients the axillary status was confirmed cytologically or histologically. 1,737 eligible patients from 103 institutions entered the trial. The $\mathrm{SN}$ detection and removal rate was $99.1 \%$ in clinically node-negative patients who underwent SNB prior to NACT. A second SNB after NACT and SNB before NACT had a detection rate of only $60.8 \%$. For patients with $\mathrm{N} 1$ before NACT and N0 after NACT the detection rate of SNB was only $80.1 \%$. Taking these results into consideration, the AGO committee recommends SNB before NACT for patients with clinical and sonographical N0 (LoE 3bC; $\mathrm{AGO}+)$. For patients with $\mathrm{N} 1$ before NACT, axillary lymphonodectomy is necessary after NACT; SNB is not recommended after NACT (LoE 2bB; AGO-).

Despite the results of the SENTINA trial and the AGO recommendations, the ACOSOG Z1071 trial [19] shows a possible clinical way to reduce the morbidity of axillary dissection for patients planned for NACT. 756 patients were enrolled in this study. Core biopsy or fine needle aspiration of the clinically and sonographically suspicious lymph node before the planned NACT was mandatory. Only patients with histologically or cytologically proven lymph node metastasis were included in the trial. pCR of the lymph node metastases was $40 \%$. After NACT, an SNB was performed. The falsenegative rate was only $7.4 \%$. If more than $2 \mathrm{SN}$ were detected and removed, the lymph node metastasis was marked with a clip after the biopsy, and radiocolloid and additional blue dye were used for SN identification.

For risk evaluation after NACT and selection of patients for adjuvant radiotherapy it is important to know the pathological lymph node status (ypNsn) after but not before the NACT [20]. The ACOSOG trial Z1071 may become the basis for the clinical management of axillary lymph node metastases in the case of planned NACT.

\section{Oncoplastic and Reconstructive Surgery}

Due to the importance of aesthetic aspects in oncologic and reconstructive breast surgery, plastic surgical techniques are indispensable in preserving the original breast contour or even in changing it or, on the other hand if not feasible, in reconstructing an aesthetically pleasing breast shape.

Oncoplastic surgery was introduced into the primary surgical treatment of breast cancer to enable wider resections in BCS with equally aesthetic results ( $\mathrm{LoE} 2 \mathrm{bB} ; \mathrm{AGO}++)$. In about $20 \%$ of cases, BCS is desired but the tumour/breast ratio (TBR) or the location of the tumour are unfavourable so that additional oncoplastic procedures such as mastopexy, tumour-adapted reduction mammaplasty or volume replacement techniques with flaps, local or distant, are needed [21]. In the case of an unfavourable TBR, NACT might improve the rate of BCS if adjuvant chemotherapy is indicated $(\mathrm{AGO}+)$ [22]. On the other hand, the surgeon should not get carried away by the possibilities of oncoplastic surgery to create wider margins; in the case of low risk $(\mathrm{ER}+$ / progesterone receptor (PR)+/HER2-) or high risk (TN) subgroups, more extensive surgery does not necessarily improve outcomes [23].

If a mastectomy is necessary, a skin-sparing or nipplesparing mastectomy offers the best options for immediate 
reconstruction with natural looking aesthetic results and sufficient oncological safety. A 2010 meta-analysis comparing 9 studies with 3,739 patients undergoing conventional mastectomy without reconstruction or skin-sparing mastectomy with reconstruction showed no significant differences in terms of local recurrence rate [24] ( $\mathrm{LoE} 2 \mathrm{bB} ; \mathrm{AGO}++)$. There are different options possible to approach the breast gland for mastectomy; the periareolar access used to be very popular but proved to bear a higher risk of necrosis. In comparison, the inferior lateral approach or the incision in the inframammary fold have shown the lowest incidence of complications.

In the case of delayed-immediate reconstruction with tissue expanders and the necessity of postmastectomy radiotherapy (PMRT), it seems advantageous to irradiate the reconstructed breast containing a permanent implant instead of an expander [25]. On the other hand, after PMRT, the delay of expanderimplant exchange by at least 6 months after completed radiotherapy can significantly reduce expander-implant failure [26] (LoE 3bC; AGO+).

With the increasing number of immediate implant reconstructions after skin-sparing mastectomies, fold formation due to the inserted silicone breast implant and irregularities in the breast surface are also seen more often. This calls for an improvement of implant coverage after mastectomy and during reconstruction. Besides the use of form-stable anatomical implants, 4 additional techniques or new materials can be helpful: autologous tissue, i.e. the endoscopically harvested latissimus dorsi flap (LoE 3bC; $\mathrm{AGO}+$ ), use of acellular dermal matrix (ADM) (LoE 3aB; $\mathrm{AGO}+$ ), use of synthetic mesh, and/or use of lipofilling ( $\mathrm{LoE}$ $3 \mathrm{bC} ; \mathrm{AGO}+)$. The rate of complications due to ADM decreases with more experience. See in this context various retrospective observational studies by experienced users like Salzberg [27] with 10-year results using ADM in direct-toimplant breast reconstructions demonstrating the effectiveness and long-term safety of the procedure. In competent hands, lipofilling is very effective and has a very low complication rate; up to date there is no proof that the procedure causes any increased risk of local recurrence. The analysis by Seth et al. [28] states that fat grafting after breast reconstruction does not adversely affect local tumour recurrence or long-term survival follow-up.

\section{Adjuvant Endocrine Treatment in Pre- and Postmenopausal Patients}

Recent data from large prospective randomized trials showed that patients with HR-positive tumours may have a small but significant advantage from prolonged endocrine therapy [29]. Therefore, the AGO Breast Committee is currently recommending a treatment duration with TAM of up to 10 years, if feasible. Current trials are underway comparing 10 vs. 15 years of treatment.

Given the small absolute differences that were observed for various approaches to give tamoxifen and/or aromatase inhibitors alone or in sequence in postmenopausal patients, the committee felt that it is more important to motivate patients to comply with treatment at full dose for the whole treatment period than to stick to one of the endocrine approaches. It is preferred to switch to another endocrine treatment in the case of toxicities impairing quality of life instead of stopping therapy or to lose the compliance of the patient. In patients with high risk of early relapse (e.g. nodepositive disease) or in patients with lobular invasive cancers, aromatase inhibitors have shown the largest benefit compared to tamoxifen and should therefore be considered as first treatment approach [30].

In premenopausal patients, the standard endocrine therapy is tamoxifen. The use of gonadotropin-releasing hormone $(\mathrm{GnRH})$ analogues without chemotherapy is considered as an option for young patients with low or intermediate risk of relapse and relevant contraindications against tamoxifen, who do not receive adjuvant chemotherapy. The addition of GnRH analogues to tamoxifen after chemotherapy is not supported by the literature [31]. As only 81 patients with age $\leq 40$ years at diagnosis have been randomized to chemotherapy+tamoxifen versus chemotherapy+tamoxifen+GnRH analogue, this approach is no longer recommended by the committee. Treatment duration of tamoxifen can be prolonged up to 10 years. There are no data for AI treatment longer than 5 years.

\section{Adjuvant Cytotoxic and Targeted Therapy}

Recently published trials and meta-analyses actually confirmed the AGO treatment recommendations with regard to adjuvant cytotoxic and targeted therapy. There were only upgrades in the LoE but no clinically relevant modifications. In the following, the main strategies in adjuvant therapy of early breast cancer are summarized.

Provided that the indication for adjuvant chemotherapy is given, anthracycline-based combination chemotherapy is regarded as minimum standard treatment (LoE 1aA; $\mathrm{AGO}++)$. As shown in a meta-analysis, these regimens provide an additional reduction in relapse rate (ratio 0.89 , $\mathrm{p}=0.0001$ ) and mortality (ratio 0.84, $\mathrm{p}<0.001$ ) compared with adjuvant CMF therapy (cyclophosphamide, methotrexate, 5-fluorouracil) after 15 years [32.]. In general, by adding taxanes, disease-free survival (DFS) and overall survival can be further improved (LoE $1 \mathrm{aA} ; \mathrm{AGO}++$ ), and eventually side effects, particularly cardiotoxicity, can be reduced. In trials adding 4 separate cycles of a taxane to a fixed anthracycline-based control regimen, breast cancer 
mortality was further reduced ( $R R 0.86, p=0.0005)$ [32]. A meta-analysis revealed significant differences in favour of the sequential regimens as compared to anthracycline-taxanecontaining combination regimens [33].

In node-positive disease, dose-dense (q2w) adjuvant chemotherapeutic schedules may improve the relapse-free and overall survival compared to conventional adjuvant treatment options (LoE $1 \mathrm{aA} ; \mathrm{AGO}++$ ). High-dose chemotherapy with autologous stem cell support is not recommended as adjuvant treatment in breast cancer (LoE 1aA; AGO-). The efficacy of CMF versus no chemotherapy has been shown in numerous trials with a long-term follow-up of now up to 20 years, and has been proven in meta-analyses [32]. Therefore, 6 cycles of CMF can be given in patients with contraindications for anthracycline- and taxane-containing regimens. Still, in patients with a risk profile indicating a need for adjuvant chemotherapy, giving $\mathrm{CMF}$ is better than giving no therapy (LoE $1 \mathrm{aA} ; \mathrm{AGO}++$ ).

Capecitabine, gemcitabine, and platinum compounds have been investigated in adjuvant trials. So far, none of these drugs can be recommended to be included into anthracycline-/ taxane-based regimens. With regard to capecitabine, several trials could not demonstrate an additional benefit when adding capecitabine to an anthracycline-/taxane-based therapy (LoE $1 \mathrm{aB} ; \mathrm{AGO}+/-)$. Most of the trials used lower doses of either the taxane or capecitabine in order to cope with toxicity.

Considering targeted therapy, all studies that demonstrated a benefit for adjuvant trastuzumab in women with HER2positive tumours included node-negative and node-positive patients, and subgroup analysis showed a benefit for both groups of patients. Therefore, trastuzumab-containing regimens can be used in node-positive ( $\mathrm{LoE} 1 \mathrm{aA} ; \mathrm{AGO}++$ ) as well as in node-negative patients whenever chemotherapy is considered adequate. Women with node-negative disease, additional risk factors, and tumour size $>10 \mathrm{~mm}$ will profit from trastuzumab (LoE 1aA; AGO++). Moreover limited data from adjuvant studies suggests that even patients with HER2-positive tumours $<10 \mathrm{~mm}$ may gain benefit from trastuzumab. Thus, the use of trastuzumab may also be considered in smaller tumours (for tumours > 5-10 mm, $\mathrm{AGO}+$; for tumours $\leq 5 \mathrm{~mm}, \mathrm{AGO}+-$-). In contrast to primary systemic therapy, the phase III trials in adjuvant therapy with other targeted agents/dual HER2 blockade such as lapatinib and pertuzumab are not yet mature (recommendation level for these 2 compounds: LoE 5D; AGO-). When lapatinib was given in delayed adjuvant treatment, DFS did not improve significantly (LoE 1bB; AGO-). The BEATRICE trial demonstrated no statistically significant improvement in invasive DFS with the addition of bevacizumab to adjuvant chemotherapy for triple-negative breast cancer (LoE 1bB; AGO-/-) [34].

\section{Adjuvant Bisphosphonates}

Several randomized phase III trials have investigated the role of bone-modifying therapy with bisphosphonates in the adjuvant setting. Data of these trials on whether bisphosphonates in addition to adjuvant treatment may improve patients' outcome are conflicting with contradictory results leaving this important issue still unanswered. Subset analyses of some trials (AZURE, NSABP-B34) suggest that the effect of adjuvant bisphosphonates is mainly confined to postmenopausal ( $\geq 50$ years, NSABP B34 trial) patients. In premenopausal patients of the AZURE trial, non-skeletal distant and locoregional recurrence was significantly higher in patients treated additionally with zoledronic acid. Based on these conflicting data, the use of bisphosphonates as adjuvant treatment is not standard of care for patients in general and may be considered in postmenopausal patients only (AGO+).

\section{Adjuvant Radiotherapy}

Over more than 3 decades, BCS followed by external beam whole-breast irradiation (WBI) was established as standard of care for early breast cancer. WBI traditionally comprises 5 weeks (50 Gy in 25 fractions), not including a possible local boost irradiation of the tumour bed following WBI. This concept has been challenged by approaches focussing on more convenient treatment providing a lower total radiation dose given in fewer slightly larger fractions and delivered over a shorter period of time by use of hypofractionation schedules [35-37]. In addition, the necessity of applying WBI in all patients was questioned by the idea of partial breast irradiation (PBI) [38-41]. The combination of these techniques, accelerated partial breast irradiation (APBI), was also subject of recently published studies [42].

The 10-year follow-up results for the UK START trials (Standardisation of Breast Radiotherapy) were presented at the 2012 San Antonio Breast Cancer Symposium [35]. Between 1999 and 2002, 4,451 women with completely excised invasive breast cancer (T1-3, N0-1, M0) were randomised after primary surgery to 3 different techniques of WBI. This quality-controlled phase III study revealed that appropriatelydosed hypofractionated radiotherapy is a safe and effective option for patients with early breast cancer. It was shown that 41.6 Gy applied in 13 fractions (experimental arm 1) and $40 \mathrm{~Gy}$ in 15 fractions (experimental arm 2) each appear to be comparable to $50 \mathrm{~Gy}$ in 25 fractions (standard arm). These findings were obvious in terms of locoregional tumour control and late normal tissue effects. Consequently, the British National Institute for Health and Clinical Excellence (NICE) introduced the hypofractionated radiotherapy (experimental arms 1 and 2) in its guidelines. The concept of hypofractionation was also proven to be safe and effective in a Canadian 
large population-based series after 10 years of follow-up for patients presenting with grade 3 breast cancer [36].

Taken together, the long-term data of these mature, large clinical trials confirm the observations of earlier studies. Consequently, AGO considers hypofractionated radiotherapy as an equal alternative approach ( $\mathrm{LoE} 1 \mathrm{aA} ; \mathrm{AGO}++)$.

\section{Breast Cancer in Specific Situations}

Although breast cancer is a relatively common disease, in specific situation, the treatments are not standardized and cancer-related deaths are higher. Patients younger than 35 years have a more aggressive tumour biology with high mortality rates. They furthermore may be interested in fertility counselling. Surgery and systemic treatment do not differ from that of early breast cancer in other age groups. Available evidence suggests that patients may benefit from tamoxifen concerning recurrence and overall survival. Therefore, tamoxifen must be offered over a period of 5-10 years (LoE 1bB; $\mathrm{AGO}++)$. GnRH agonists during chemotherapy treatment appear to protect against chemotherapy-related premature ovarian failure in the first year after treatment. According to current data, they have no effect on the resumption of menses or spontaneous pregnancy rates. Therefore, their use is not recommended for fertility preservation (LoE 1aA; AGO-). Fertility counselling before systemic treatment is mandatory.

Elderly patients over 65 years represent about $50 \%$ of breast cancer patients, and their number will rise in the future. Today no aspect of the management of older women with breast cancer should be driven by chronological age. Geriatric assessment and treatment according to standard are recommended (LoE 2bB; AGO++). Patients should be closely monitored for side effects with prompt intervention. Standard treatment must be reduced in frail patients with substantial comorbidity or if life expectancy is less than 5 years (LoE 2bB; $\mathrm{AGO}++$ ).

Diagnostics, surgery and systemic treatment in male breast cancer do not differ from that in women. Screening for 2 nd malignancies and genetic counselling are recommended $(\mathrm{AGO}++)$.
Cancer of unknown primary (CUP) represents about 5\% of advanced cancers per year. Therapy for the majority of patients in the absence of a specific tumour diagnosis has been empiric and relatively ineffective. ER/PR/HER2 as surrogate markers for molecular subtypes $(\mathrm{AGO}++)$ and molecular profiling of tumours are promising techniques to reveal the primary site in CUP patients ( $\mathrm{LoE} 2 \mathrm{cB} ; \mathrm{AGO}+-$ ). Systemic treatment must be according to the treatment in $\mathrm{N}+$ patients (LoE 3aC; $\mathrm{AGO}++$ ).

Complete local excision with clear margins is the most important therapy in the very aggressive angiosarcoma and malignant phyllodes tumour ( $\mathrm{LoE} 3 \mathrm{aC}$; $\mathrm{AGO}++$ ). Axillary node dissection is not recommended if lymph nodes are not involved clinically (AGO-).

\section{Hormonal Treatment and Alternatives in Breast Cancer Survivors}

Menopausal symptoms in young patients with breast cancer are typically more severe due to the abrupt and rapid decrease in oestrogen, and chemotherapy and endocrine treatment worsen these symptoms. Evidence that complementary therapies are useful is scarce for the majority of them. Behavioural modification and yoga may be helpful in mild cases of vasomotor symptoms, whereas newer antidepressants are promising in moderate to severe cases. Local vaginal moisturizers, and in refractory cases, low-dose oestrogen creams (estriol), may ameliorate most urogenital symptoms. At present, there is insufficient evidence to support any natural agent as a viable alternative to hormone therapy to treat these symptoms. But the data for the use of hormone therapy of postmenopausal symptoms is also controversial $[43,44]$. Acupuncture may be an option for these women. Data from randomized trials shows a significant effect in reducing menopausal symptoms [45, 46]. In summary, no single agent can ameliorate menopausal symptoms in breast cancer survivors. Further study is needed to identify safe and effective treatments for menopausal symptoms and to confirm their long-term safety in young breast cancer survivors.

\section{References}

1 CEBM: www.cebm.net/index.aspx?o $=1025$ (download 20-5-13).

2 AGO Kommission Mamma: www.ago-online.de/de/ fuer-mediziner/leitlinien/mamma/.

3 Hammond MEH, Hayes DF, Dowsett M, et al. American Society of Clinical Oncology/College of American Pathologists guideline recommendations for immunohistochemical testing of estrogen and progesterone receptors in breast cancer. J Clin Oncol 2010;28:2784-2795.
4 Wolff AC, Hammond MEH, Schwartz JN, et al.: American Society of Clinical Oncology/College of American Pathologists guideline recommendations for human epidermal growth factor receptor 2 testing in breast cancer. J Clin Oncol 2007;25:118145.

5 Kennecke HF, Speers CH, Ennis CA, Gelmon K, Olivotto IA, Hayes M: Impact of routine pathology review on treatment for node-negative breast cancer. J Clin Oncol 2012;30:2227-2231.
6 Vestjens JHMJ, Pepels MJ, et al.: Relevant impact of central pathology review on nodal classification in individual breast cancer patients. Ann Oncol 2012;23:2561-2566.

7 Simon RM, Paik S, Hayes DF: Use of archived specimens in evaluation of prognostic and predictive biomarkers. J Natl Cancer Inst 2009; 101:1446-1452.

8 Paik S, Shak S, Tang G, et al.: A multigene assay to predict recurrence of tamoxifen-treated, nodenegative breast cancer. N Engl J Med 2004;351: 2817-2826. 
9 Paik S, Tang G, Shak S, et al.: Gene expression and benefit of chemotherapy in women with nodenegative, estrogen receptor-positive breast cancer. J Clin Oncol 2006;24:3726-3734.

10 Filipits M, Rudas M, Jakesz R, et al.: A new molecular predictor of distant recurrence in ERpositive, HER2-negative breast cancer adds independent information to conventional clinical risk factors. Clin Cancer Res 2011;17:6012-6020.

11 Van't Veer LJ, Dai H, van de Vijver MJ, et al.: Gene expression profiling predicts clinical outcome of breast cancer. Nature 2002;415:530-536.

-12 Parker JS, Mullins M, Cheang MCU, et al.: Supervised risk predictor of breast cancer based on intrinsic subtypes. J Clin Oncol 2009;27:1160-1167.

13 Robert Koch Institut: Krebs in Deutschland 2007/2008. 8. Ausgabe. Robert Koch-Institut (Hrsg) und die Gesellschaft der epidemiologischen Krebsregister in Deutschland e.V. (Hrsg). Berlin, 2012, pp. 64.

14 Shaitelman SF, Wilkinson JB, Kestin LL, Ye H, Goldstein NS, Martinez AA, Vicini FA: Long-term outcome in patients with ductal carcinoma in situ treated with breast-conserving therapy: implications for optimal follow-up strategies. Int J Radiat Oncol Biol Phys 2012;83:e305-12.

15 Staley H, McCallum I, Bruce J: Postoperative tamoxifen for ductal carcinoma in situ. Cochrane Database Syst Rev 2012;10:CD007847.

16 Alba E, Chacon JI, Lluch A, Anton A, Estevez L, Cirauqui B, Carrasco E, Calvo L, Segui MA, Ribelles N, Alvarez R, Sanchez-Muñoz A, Sanchez R, Garcia-Asenjo JA, Rodriguez-Martin C, Escudero MJ, Albanell J: A randomized phase II trial of platinum salts in basal-like breast cancer patients in the neoadjuvant setting. Results from the GEICAM/2006-03, multicenter study. Breast Cancer Res Treat 2012;136:487-493.

17 Von Minckwitz G: Neoadjuvant chemotherapy in breast cancer-insights from the German experience Breast Cancer 2012;19:282-288.

18 Kuehn T, Bauerfeind IGP, Fehm T, Fleige B, Helms G, Lebeau A, Liedtke C, von Minckwitz G Nekljudova V, Schrenk P, Staebler A, Untch M: Sentinel lymph node biopsy before or after neoadjuvant chemotherapy - final results from the prospective german, multiinstitutional Sentinatrial. SABCS 2012, S2-2. Cancer Res 2012;72(24 suppl):89s.

19 Boughey JC, Suman VJ, Mittendorf EA, Ahrendt GM, Wilke LG, Taback B, Leitch AM Flippo-Morton TS, Byrd DR, Ollila DW, Julian TB, McLaughlin SA, McCall L, Symmans WF, Le-Petross HT, Haffty BG, Buchholz TA, Hunt KK: The role of sentinel lymph node surgery in patients presenting with node positive breast cancer (T0-T4, N1-2) who receive neoadjuvant chemotherapy - results from the ACOSOG Z1071 trial. SABCS 2012, S2-1. Cancer Res 2012;72(24 suppl):89s.

20 Mamounas EP, Anderson SJ, Dignam JJ, Bear HD, Julian TB, Geyer CE Jr, Taghian A, Wickerham DL, Wolmark N: Predictors of locoregional recurrence after neoadjuvant chemotherapy: results from combined analysis of National Surgical Adjuvant Breast and Bowel Project B-18 and B-27. J Clin Oncol 2012;30:39603966

21 Brunnert KE: The Osnabrueck experience with reconstruction of the partial mastectomy defect; in
Spear SL (ed): Surgery of the Breast, 3rd ed. Philadelphia, PA, Lippincott Williams \& Wilkins, 2011, pp. 177-197.

22 Huober J, von Minckwitz G: Neoadjuvant therapy - what have we achieved in the last 20 years? Breast Care (Basel) 2011;6:419-426.

23 Houssami N: Meta-analysis of the impact of surgical margins on local recurrence in women with early-stage invasive breast cancer treated with BCT. Eur J Cancer 2010;46:3219.

24 Lanitis S, Tekkis PP, Sgourakis G, Dimopoulos N, Al Mufti R, Hadjiminas DJ: Comparison of skinsparing mastectomy versus non-skin-sparing mastectomy for breast cancer: a meta-analysis of observational studies. Ann Surg 2010;251:632-639.

25 Nava MB, Pennati AE, Lozza L, Spano A, Zambetti M, Catanuto G: Outcome of different timings of radiotherapy in implant-based breast reconstructions. Plast Reconstr Surg 2011;128:353359.

26 Peled AW, Foster RD, Esserman LJ, Park CC, Hwang ES, Fowble B: Increasing the time to expander-implant exchange after postmastectomy radiation therapy reduces expander-implant failure. Plast Reconstr Surg 2012;130:503-509.

27 Salzberg CA: Focus on technique: one-stage implant-based breast reconstruction. Plast Reconstr Surg 2012;130(5 suppl 2):95S-103S.

28 Seth AK, Hirsch EM, Kim JY, Fine NA: Long-term outcomes following fat grafting in prosthetic breast reconstruction: a comparative analysis. Plast Reconstr Surg 2012;130:984-989.

29 Davies C, Pan H, Godwin J, Gray R, Arriagada R, Raina V, Abraham M, Alencar VH, Badran A, Bonfill X, Bradbury J, Clarke M, Collins R, Davis SR, Delmestri A, Forbes JF, Haddad P, Hou MF, Inbar M, Khaled H, Kielanowska J, Kwan WH, Mathew BS, Mittra I, Müller B, Nicolucci A, Peralta O, Pernas F, Petruzelka L, Pienkowski T, Radhika R, Rajan B, Rubach MT, Tort S, Urrútia G, Valentini M, Wang Y, Peto R; for the Adjuvant Tamoxifen: Longer Against Shorter (ATLAS) Collaborative Group: Long-term effects of continuing adjuvant tamoxifen to 10 years versus stopping at 5 years after diagnosis of oestrogen receptor-positive breast cancer: ATLAS, a randomised trial. Lancet 2012;pii:S01406736(12)61963-1.

30 Metzger O, Giobbie-Hurder A, Mallon E, et al.: Relative effectiveness of letrozole compared with tamoxifen for patients with lobular carcinoma in the BIG 1-98 trial. SABCS 2012; abstr S1-1.

31 Cuzick J, Ambroisine L, Davidson N, Jakesz R, Kaufmann M, Regan M, Sainsbury R: LHRHagonists in Early Breast Cancer Overview group. Use of luteinising-hormone-releasing hormone agonists as adjuvant treatment in premenopausal patients with hormone-receptor-positive breast cancer: a meta-analysis of individual patient data from randomised adjuvant trials. Lancet 2007; 369:1711-1723.

32 Peto R, Davies C, Godwin J, Gray R, Pan HC, Clarke M, Cutter D, Darby S, McGale P, Taylor C, Wang YC, Bergh J, Di Leo A,. Swain S, Piccart M, Pritchard; Early Breast Cancer Trialists' group (EBCTCG): Comparison between different polychemotherapy regimes for early breast cancer: meta-analyses of long-term outcome among 100,000 women in 123 randomised trials. Lancet 2012;379:432-444.
3 Shao N, Wang S, Yao C, Xu X, Thang Y, Zhang Y, Lin Y: Sequential versus concurrent anthracyclines and taxanes as adjuvant chemotherapy of early breast cancer: a meta-analysis of phase III randomized control trials. Breast 2012;21:389-393.

34 Cameron D, Brown J, Dent R, Jackisch C, Mackey J, Pivot X, Steger G, Suter T, Toi M, Parmar M, Bubuteishvili-Pacaud L, Henschel V, Laeufle R, Bell R: Primary results of BEATRICE, a randomized phase III trial evaluating adjuvant bevacizumab-containing therapy in triple-negative breast cancer. SABCS 2012; abstr S6-5.

35 Haviland JS, Agrawal R, Aird E, et al., on behalf of the START Trialists: The UK START (Standardisation of Breast Radiotherapy) trials: 10year follow-up results. SABCS 2012;abstr S4-1.

36 Herbert C, Nichol A, Olivotto I, et al.: The impact of hypofractionated whole breast radiotherapy on local relapse in patients with grade 3 early breas cancer: a population-based cohort study. Int J Radiat Oncol Biol Phys 2012;82:2086-2092.

37 James ML, Lehman M, Hider PN, et al.: Fraction size in radiation treatment for breast conservation in early breast cancer. Cochrane Database Syst Rev 2010;CD003860

38 Njeh CF, Saunders MW, Langton CM: Accelerated partial breast irradiation using external beam conformal radiation therapy: a review. Crit Rev Oncol Hematol 2012;81:1-20.

39 Hattangadi JA, Taback N, Neville BA, Harris JR, Punglia RS: Accelerated partial breast irradiation using brachytherapy for breast cancer: patterns in utilization and guideline concordance. J Natl Cancer Inst 2012;104:29-41.

40 Vaidya JS, Wenz F, Bulsara M, et al.: Targeted intraoperative radiotherapy for early breast cancer: TARGIT-A trial - updated analysis of local recurrence and first analysis of survival. SABCS 2012; abstr S4-2.

41 Bartelink H, Bourgier C, Elkhuizen P: Has partial breast irradiation by IORT or brachytherapy been prematurely introduced into the clinic? Radiother Oncol 2012;104:139-142.

42 Freedman GM, Anderson PR, Bleicher RJ, et al.: Five-year local control in a phase II study of hypofractionated intensity modulated radiation therapy with an incorporated boost for early stage breast cancer. Int $\mathrm{J}$ Radiat Oncol Biol Phys 2012;84:888-893.

43 Holmberg L, Anderson H; HABITS steering and data monitoring committees: HABITS (hormonal replacement therapy after breast cancer - is it safe?), a randomised comparison: trial stopped. Lancet 2004;363:453-455.

44 Fahlén M, Fornander T, Johansson H, Johansson U, Rutqvist LE, Wilking N, von Schoultz E: Hormone replacement therapy after breast cancer: 10 year follow up of the Stockholm randomised trial. Eur J Cancer 2013;49:52-59.

45 Bokmand S, Flyger H: Acupuncture relieves menopausal discomfort in breast cancer patients: a prospective, double blinded, randomized study. Breast 2013;22:320-323.

46 Molassiotis A, Bardy J, Finnegan-John J, Mackereth P, Ryder DW, Filshie J, Ream E, Richardson A: Acupuncture for cancer-related fatigue in patients with breast cancer: a pragmatic randomized controlled trial. J Clin Oncol 2012; 30:4470-4476. 